\title{
Incremental Validity of Mindfulness-Based Attention in Relation to the Concurrent Prediction of Anxiety and Depressive Symptomatology and Perceptions of Health
}

\author{
Michael J. Zvolensky, Sondra E. Solomon, Alison C. McLeish, Daniel Cassidy, \\ Amit Bernstein, Carrie J. Bowman and Andrew R. Yartz \\ Department of Psychology, University of Vermont, Burlington, Vermont, USA
}

\begin{abstract}
This investigation evaluated the role of mindfulness-based attention in concurrently predicting anxiety and depressive symptomatology and perceived health functioning in a community sample of 170 young adults ( 95 females; mean age $\left(\mathrm{M}_{\text {age }}\right)=22.2$ years, $\left.\mathrm{SD}=7.6\right)$. Partially consistent with prediction, results indicated that, relative to negative and positive affectivity and emotional expression and processing associated with approach-oriented coping, mindfulness-based attention incrementally predicted anhedonic depressive, but not anxious arousal, symptoms. Additionally, consistent with prediction, mindfulness-based attention demonstrated incremental validity in relation to perceived health, and the degree of impairment of health in terms of physical and mental functioning. Results are discussed in relation to the construct development of mindfulness-based attention, and specifically, the role(s) of this factor in emotional and physical health processes. Key words: mindfulness; attention; perceived health; anxiety; depression; positive and negative affectivity; approach-oriented coping.
\end{abstract}

Received December 28, 2005; Accepted March 6, 2006

Correspondence address: Michael J. Zvolensky, PhD, The University of Vermont, Department of Psychology, 2 Colchester Avenue, John Dewey Hall, Burlington, VT 05405-0134, USA. Tel: +802 656 8994; Fax:+802656 8783. E-mail: Michael.Zvolensky@uvm.edu

There has been increased scientific and clinical attention focused on better understanding the role of mindfulness in psychological and physical health problems (Baer, Smith, \& Allen, 2004; Bishop et al., 2004; Buchheld, Grossman, \& Walach, 2002; Conte, Plutchik, Jung, \& Picard, 1990; Demick, 2000). Such interest has broadly grown from the recognition that there are potential emotional and physical health benefits to mindfulness defined from a number of distinct perspectives (Langer, 1989; Langer \& Moldoveneau, 2000; Wilber, 2000; see Zvolensky, Feldner, LeenFeldner, \& Yartz, 2005, for a review).

Brown and Ryan (2003), in particular, have developed a promising and theoreticallyderived self-report measure entitled the Mindful Attention Awareness Scale (MAAS), which assesses individual differences in the frequency of mindful states over time. The conceptual basis of the MAAS is steeped broadly in self-regulatory models of psychological functioning and self-awareness. According to Brown and Ryan (2003), mindfulness-based attention, as indexed by the MAAS, denotes conscious "attention to, and awareness of, what is occurring in the present moment" (p. 824). For the purposes of the present paper, hereafter we refer to this particular definition of mindfulness as "mindfulness-based attention" to distinguish it from alternative perspectives. This construct differs theoretically and empirically from other selfregulatory and self-awareness constructs, such as openness to experience, knowledge of self, and emotional intelligence (Brown \& Ryan, 2003, see e.g. Table 3). Though limited in overall scope, available research on the 
MAAS suggests that there is meaningful between-person and within-person variability in mindfulness-based attention, and moreover, that such variability is related to positive emotional states and self-regulatory behaviour. For example, the MAAS total score is concurrently positively related to constructs such as "openness to experience" and "internal state awareness" (Brown \& Ryan, 2003, Study 1). Other data indicate that mindfulness-based attention is negatively related to the intensity and frequency of negative affect symptoms over time (Brown \& Ryan, 2003, Study 4). These data, in conjunction with contemporary theoretical models (Bishop et al., 2004), suggest that this attention-based conceptualization of mindfulness may hold promise in terms of understanding psychological vulnerability and resiliency for emotional problems and health behaviours. Although existing work on mindfulness-based attention is indeed encouraging, there are presently at least $3 \mathrm{key}$ issues to address in future study of this construct.

A primary area in need of future scientific attention centres on evaluating whether mindfulness-based attention offers unique explanatory power relative to other well-established risk and protective factors, namely negative and positive affectivity (Watson, Clark, \& Tellegen, 1988). These temperamental factors related to a generalized, largely inherited, tendency to experience positive and negative affect are formatively associated with psychological functioning including mood states and coping or self-regulatory processes related to adapting to such states (e.g. Gray \& McNaughton, 1996; Watson, David, \& Suls, 1999). Thus, to the extent mindfulness-based attention, as measured by the MAAS, reflect distinct self-regulatory processes, negative and positive affectivity may share important associations with these variables. To date, there has been only 1 test of the unique explanatory value or incremental validity of mindfulnessbased attention relative to the theoreticallyrelevant constructs of negative and positive affectivity (Brown \& Ryan, 2003). Results from that work indicated that MAAS total scores accounted for unique variance in anxiety and depressive symptoms after controlling for the singular effects of either negative affectivity or positive affectivity (Brown \& Ryan, 2003, see Table 5).
However, it is noteworthy that these temperamental factors (positive and negative affectivity) were not included together as covariates in the analytic model (i.e. entered simultaneously). Thus, the observed effects may potentially be attributed to shared variability of mindfulness-based attention with the alternative (uncontrolled) affectivity factor (i.e. one needs to consider both, rather than 1 , of these factors in tests of mindfulness-based attention). In other words, individuals high in mindfulness-based attention may be prone to experience positive affect or not vulnerable to negative affect. Similarly, individuals low in mindfulness-based attention may be prone to greater levels of negative affect or low levels of positive affect. To aid the construct development of mindfulness-based attention, a more stringent test of incremental validity of mindfulness-based attention is needed to explicate the unique variance accounted for by the construct in anxiety and depressive symptoms after simultaneously controlling for both negative and positive affectivity.

A second key issue in need of further research on mindfulness-based attention pertains to distinguishing the construct from theoretically relevant self-regulation processes, and specifically, approach-oriented coping variables. Research suggests that there are at least 2 distinct forms of emotional approach coping, reflecting adaptation to life events through emotional (i) expression (i.e. time and effort given to the expression of affective states) and (ii) processing (i.e. active attempts to acknowledge, and understand one's emotions; Stanton, Kirk, Cameron, \& Danoff-Burg, 2000). These approach-oriented coping constructs are theoretically related to the regulation of attention focused on interoceptive (e.g. emotional states) and exteroceptive (e.g. external stressors) stimuli in that they all probably involve the activation of "present-centred attention." Such approachoriented attention allocation is similar to mindfulness, which entails an "open" level of awareness and attention to present interoceptive processes and external life events (Hayes \& Wilson, 2003; Roemer \& Orsillo, 2002). Aside from this theoretical similarity, it is noteworthy that emotional expression and processing factors also are empirically related to outcomes in a manner similar to mindfulness-based attention. For instance, similar 
to mindfulness-based attention (Brown \& Ryan, 2003), emotional expression and processing are both positively related to enhanced emotional adjustment to specific aversive life events (Smyth \& Pennebaker, 1999), as well as decreased global levels of the frequency and intensity of negative emotional states (Stanton et al., 2000). Thus, to determine whether mindfulness-based attention is uniquely important for understanding emotional vulnerability processes, it is important to test whether mindfulness-based attention is distinguishable and thereby accounts for unique variance over and above established and theoretically-relevant indices of approachoriented coping in the prediction of negative emotional states.

A third issue in need of further empirical study pertains to the relative specificity of mindfulness effects vis-à-vis aspects of psychological and physical functioning. Although there has been longstanding interest in mindfulness in regard to psychological functioning (Roemer \& Orsillo, 2002; Williams, Teasdale, Segal, \& Soulsby, 2000), mindfulness also holds relevance in relation to various facets of physical health (Kabat-Zinn, Lipworth, Burney, \& Sellers, 1986). One promising, yet currently unstudied, aspect of research in this domain would be to explore associations between mindfulness-based attention and subjective perceptions of physical health. Perceived health, broadly defined as beliefs about one's health status/quality (Mossey \& Shapiro, 1982), is related to key aspects of life functioning including mortality (Mossey \& Shapiro, 1982), healthcare-seeking behaviour even after controlling for actual physical health status (Idler \& Angel, 1990; Idler \& Kasl, 1991) and health-related fear and anxiety (Schmidt, Joiner, Staab, \& Williams, 2003; Schmidt, Telch, \& Joiner, 1996). Drawing from existing indirect work, mindfulness-based attention may be related to perceived health and the degree to which perceptions of one's health may impact mental and physical aspects of functioning. For example, mindfulness-based attention to health-related symptoms and factors may increase the probability that somatic events are attended to rather than avoided. Thus, mindfulness may prompt more adaptive health behaviour by virtue of being psychologically "in contact" with, or exposed to, internal states (Reibel, Greeson, Brainard, \& Rosenzweig, 2001). Building on both theory and past empirical work, it is therefore important to evaluate empirically the unique explanatory value of mindfulness-based attention in relation to perceived health status and health impairment, after controlling for variance related to affect-relevant temperamental and approach-oriented coping factors.

Together, the overarching aim of the present investigation was to concurrently examine the incremental validity of mindfulness-based attention beyond other theoretically-relevant risk and protective factors in terms of anxiety and depressive symptoms and perceived health variables. To be considered a unique explanatory factor related to differential risk for anxiety symptoms, depressive symptoms, or health impairment, mindfulness-based attention would need to demonstrate unique predictive value beyond that accounted for by the conceptually-related variables of negative and positive affectivity (simultaneously), as well as the established approach coping factors of emotional expression and processing. Based upon past research and theory on mindfulness (Roemer \& Orsillo, 2002), it was hypothesized that mindfulness-based attention would offer unique explanatory value relative to these theoretically-relevant covariates for both: (i) anxiety and depressive symptoms; and (ii) perceived global health, as well as the perceived impact of health on physical and mental functioning. Due to the conceptual similarity between mindfulness-based attention and the strategically based covariates, which is necessary for the rigorous test of its incremental validity, we also expected that the observed effects would be small to medium in size.

\section{Method}

\section{Participants}

The sample consisted of 170 young adults (95 females; mean age $\left(\mathrm{M}_{\text {age }}\right)=22.2$ years, $\mathrm{SD}=7.6)$ recruited through the general community in Vermont via advertising using flyers displayed in a local well-travelled marketplace, local restaurants, bars, and universitybased bulletin boards. The racial composition of the studied sample reflected that of the local population (State of Vermont Department of Health, 2000): approximately $94 \%$ of the sample was Caucasian, $2.4 \%$ 
African-American, $\quad 1.2 \%$ Hispanic, $1.2 \%$ Asian American and $1.2 \%$ other. Approximately $4.8 \%$ of the sample had at least a 4-year college education, $52.4 \%$ had some college education, $39.4 \%$ had a high school degree or the equivalent, $2.9 \%$ did not have a high school education, and $0.6 \%$ did not respond to this item. The participants reported the following lifetime history of medical problems: $8.23 \%$ had experienced some type of head injury, $3.53 \%$ had been diagnosed with heart problems, $2.35 \%$ had been diagnosed with hypertension, $16.47 \%$ had allergies, $4.12 \%$ had been diagnosed with asthma, $1.18 \%$ had some other form of respiratory disease, and $0.59 \%$ had epilepsy. Participants were excluded from the study if they displayed limited mental competency or the inability to give informed, written consent.

\section{Measures}

Mindful Attention Awareness Scale $(M A A S)$. The MAAS is a 15 -item questionnaire in which respondents indicate, on a 6point Likert-type scale $(1=$ almost always to $6=$ almost never), their level of awareness and attention to present events and experiences (Brown \& Ryan, 2003). Sample MAAS items include "I rush through activities without being really attentive to them" and "I find it difficult to stay focused on what's happening in the present." A mean rating score is calculated with higher scores indicating greater mindfulness. The MAAS shows good internal consistency across a wide range of samples $(\alpha=0.80-0.87$; Brown \& Ryan, 2003). The MAAS also has been shown to be negatively correlated with measures of depression and anxiety and positively correlated with measures of positive affect and selfesteem (Brown \& Ryan, 2003; see Table 3). Test-retest reliability data over a 1-month time period suggest mindfulness-based attention, as indexed by the MAAS, is stable (i.e. no significant differences in MAAS scores between time 1 and time 2; Brown \& Ryan, 2003).

Mood and Anxiety Symptom Questionnaire (MASQ). The MASQ is a comprehensive measure of affective symptoms with wellestablished psychometric properties (see Watson et al., 1995, for details). Participants indicate how much they have experienced each symptom from 1 (not at all) to 5 (extremely). The General Distress: Depressive Symptoms scale (MASQ: GDD) measures depressed mood expected to be nondifferentiating relative to anxiety (e.g. "felt discouraged"). The General Distress: Anxious Symptoms scale (MASQ: GDA) indexes anxious mood expected to be non-differentiating relative to depression (e.g. "felt nervous"). The Anxious Arousal scale (MASQ-AA) measures the symptoms of somatic tension and arousal (e.g. "felt dizzy"). The Anhedonic Depression scale (MASQ-AD) measures a loss of interest in life (e.g. "felt nothing was enjoyable") and reverse-keyed items measuring positive affect. As in past work (Zvolensky, Kotov, Antipova, \& Schmidt, 2005), only the MASQ-AA and MASQ-AD subscales were used in the present investigation, as they provide empirically sound and specific composites for "pure" anxiety and "pure" depression symptoms, respectively (Watson et al., 1995). The alpha for the anxious arousal scale in the present sample was 0.91 and the anhedonic depressive subscale was 0.89 . In the present investigation, these subscales, specifically, allow for tests of specificity between mindfulness-based attention and the unique symptoms of anxiety and depressive states. In this way, the MASQ can provide a degree of precision not permissible by other measures of anxiety and depressive symptoms.

Short Form General Health Survey (GHS). The short-form General Health Survey is a 20 -item questionnaire in which respondents indicate on Likert-type scales perceptions of their own health status, impact of health on their physical and mental health functioning, and functional limitations attributed to that health status (Stewart, Hays, \& Ware, 1988). The GHS has demonstrated adequate reliability and validity (Gregor, Zvolensky, Leen-Feldner, Yartz, \& Feldner, in press; Gregor, Zvolensky, \& Yartz, 2005; Schmidt \& Telch, 1997; Stewart et al., 1988; Yartz, Zvolensky, Gregor, Feldner, \& LeenFeldner, 2005). In the present study, we utilized 3 theoretically relevant subscales of the GHS. The first was the perceived physical health subscale of the GHS (PGH). The PGH consists of 5 items (2 reverse-scored) which are converted to 100-point scales and averaged to a single score, with low values indicating poor 
health perceptions and higher scores indicating better perceived health. Sample items from the PGH subscale include "In general, would you say your health is..." rated on a 5-point Likert scale $(1=$ excellent to $5=$ poor $)$, as well as "I am somewhat ill" and "I am healthy" rated on a 5-point Likert scales $(1=$ definitely true to $5=$ definitely false). The second was the perceived impact of health on physical functioning subscale of the GHS (PF). The PF consists of 6 items that are converted to 100point scales and averaged to a single score, with low values indicating poor physical functioning and higher scores indicating better physical functioning (i.e. fewer limitations). Sample items from the PF subscale include "For how long (if at all) has your health limited you in running or participating in strenuous sports?" and "For how long (if at all) has your health limited you in bending, lifting, or stooping?" rated on a 3-point Likert scale $(1=$ limited for more than 3 months to $3=$ not limited at all). The third subscale of the GHS employed in the present study was the perceived impact of health on mental functioning $(\mathrm{MH})$. The $\mathrm{MH}$ consists of 5 items (2 reverse-scored) that are converted to 100point scales and averaged to a single score, with low values indicating poor mental functioning and higher scores indicating better mental functioning. Sample items from the $\mathrm{MH}$ subscale include "How much of the time during the past month have you felt downhearted and blue?" and "How much of the time during the past month have you felt calm and peaceful?"' rated on a 3-point Likert scale $(1=$ all of the time to $3=$ none of the time).

Positive Affect Negative Affect Schedule ( $P A N A S)$. The PANAS is a mood measure commonly used in psychopathology research (Watson, Clark, \& Tellegen, 1988). For each of 20 adjectives, participants indicate on a 5point Likert-type scale the degree to which the descriptor reflects how they generally feel. It assesses 2 global dimensions of affect: negative and positive. Both the positive affect scale (PANAS-PA) and the negative affect scale (PANAS-NA) were used in this study. A large body of literature supports reliability and validity of the PANAS (Watson, 2000).

Emotional Approach Coping Questionnaire $(E A C Q)$. The EACQ is an 8-item questionnaire in which respondents indicate, on a 4-point Likert-type scale ( $1=\mathrm{I}$ usually don't do this at all to $4=\mathrm{I}$ usually do this a lot), their tendency to approach their emotions in response to stressful or difficult situations (Stanton et al., 2000). It measures 2 factors of emotional approach coping: emotional processing and emotional expression. Sample items from the emotional processing (EP) subscale include "I take time to figure out what I'm really feeling" and "I acknowledge my emotions." Sample items from the emotional expression (EE) subscale include "I let my feelings come out freely" and "I feel free to express my emotions." The emotional processing and emotional expression subscales show good internal consistency ( $\alpha=0.72$ and 0.82 , respectively) and test-retest reliability $(r=0.73$ and 0.72 , respectively; Stanton et al., 2000).

\section{Procedure}

Participants responding to community-based advertisements for the study were scheduled for an individual appointment by a trained research assistant. At this appointment, participants first were presented with a lay summary description of the study, gave verbal and written consent, and then completed a self-report battery assessing mindfulness and affect-related variables. Upon completion of the study, participants were debriefed regarding the aims of the study and compensated US\$25.

\section{Results}

\section{General data analytic strategy}

All data were entered, and then randomly checked by a second party. The data were then checked for error, such as outliers, by a third party. Finally, the data were evaluated for disproportionate skew and outliers. There were no major problems detected in this process.

Five dependent variables were utilized: 2 emotion-related dependent variables (anxious arousal and anhedonic depression, as indexed by the MASQ) and 3 perceived health-related dependent variables (perceived general health, and the perceived impact of health on physical and mental functioning, as indexed by relevant subscales from the GHS-Short-form; Stewart et al., 1988). Five total hierarchical linear regression analyses were performed, 1 
for each of the dependent measures. At level 1 in each model, positive and negative affectivity, as indexed by the PANAS, and emotional expression and emotional processing, as indexed by the EACQ, were included as covariates. These theoretically relevant factors were utilized as covariates to ensure any observed effects for mindfulness-based attention were not due to shared variance with these other variables. Mindfulness-based attention, as indexed by the MAAS, was entered into level 2 of the model. Employing this approach we ensured that any observed effects for mindfulness-based attention at level 2 in the model are unique and cannot be attributed to variance shared with variables in level 1 (Cohen \& Cohen, 1983).

\section{Descriptive data and relations among theoretically-relevant variables}

See Table 1 for means and standard deviations of the relevant predictor and criterion variables. Patterns of association between mindfulnessbased attention were first examined in relation to the other predictor variables (covariates). As expected, mindfulness-based attention was significantly positively associated with positive affectivity $(r=0.17, p<0.05)$ and negatively associated with negative affectivity $(r=-0.21, p<0.01)$. Mindfulness-based attention was not significantly related to either emotional processing $(r=0.12$, n.s.) or emotional expression $(r=0.08$, n.s.). Patterns of associations between mindfulness and the dependent measures were subsequently examined. As hypothesized, mindfulness-based attention demonstrated significant negative associations with both anxious arousal $(r=-0.21, p<0.01)$ and anhedonic depression $(r=-0.31, \quad p<0.001) . \quad$ Mindfulness-based attention also demonstrated the expected positive association with perceived general health $(r=0.25, p<0.001)$, as well as the perceived impact of health on physical $(r=0.26, p<0.001)$ and mental functioning $(r=0.27, p<0.001)$.

\section{Mindfulness-based attention in the prediction of emotion-related criteria}

For anxious arousal symptoms, negative affectivity $(p<0.001$; Step $1 \quad$ Beta $=0.60)$ accounted for a significant amount of variance $($ Step 1 Variance $=38.4 \%$ ), whereas
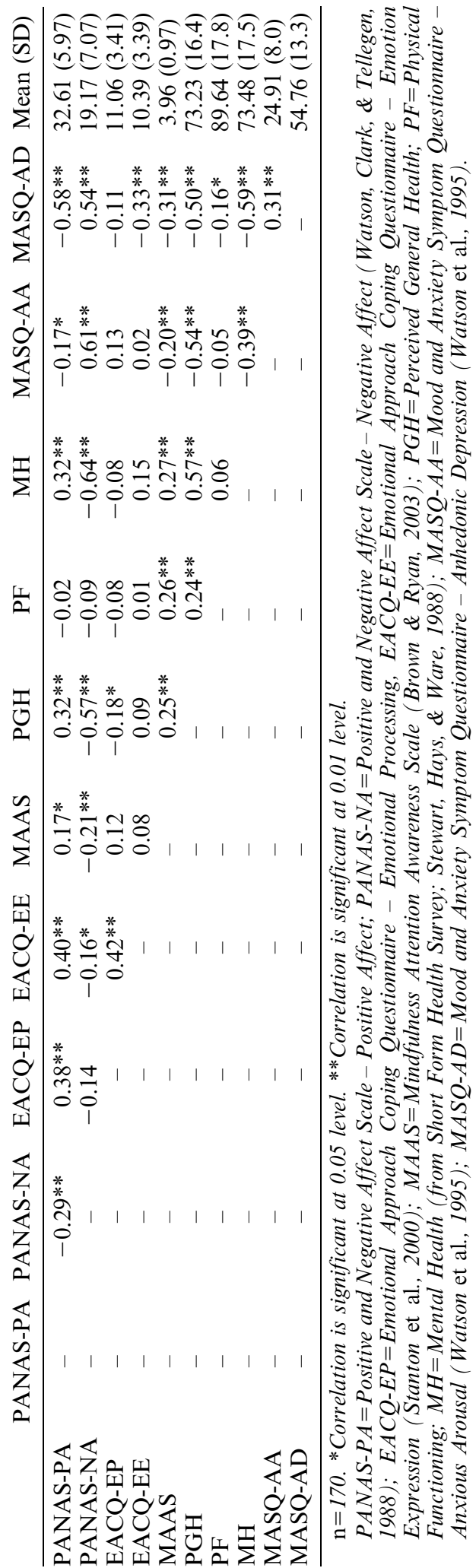
positive affectivity $(p>0.05 ; \quad$ Step 1 Beta $=-0.06)$, emotional processing $(p>0.05$; Step 1 Beta $=0.02)$, and emotional expressivity $(p>0.05$; Step 1 Beta $=0.12)$ did not. Contrary to prediction, mindfulness-based attention entered at level 2 in the model did not significantly increase the prediction of anxious arousal symptoms beyond step 1 covariates $(0.7 \%, p>0.05$; Step 2 Beta $=-0.08)$.

For anhedonic depression symptoms, positive affectivity $(p<0.001$; Step 1 ; Beta $=-0.44)$ and negative affectivity $(p<0.001$; Step 1 Beta $=0.38)$ accounted for a significant amount of variance $(49.4 \%$; $p<0.001)$, whereas emotional processing $(p>0.05$; Step 1 Beta $=0.06)$ and emotional expression $(p>0.05$; Step 1 Beta $=-0.12)$ did not. As hypothesized, mindfulness-based attention entered at level 2 in the model explained a significant amount of unique variance $\quad(2.7 \%, \quad p<0.01 ; \quad$ Step 2 Beta $=-0.17$ ), with greater levels of mindfulness predicting fewer anhedonic symptoms of depression.

\section{Mindfulness-based attention in the prediction of perceived health-related criteria}

For perceived general health, positive affectivity $(p<0.001$; Step 1 ; Beta $=0.27)$, negative affectivity $(p<0.001$; Step 1 Beta $=-0.46)$, and emotional processing $(p<0.01$; Step 1 ; Beta $=-0.22$ ) each accounted for a significant amount of variance in step $1(39 \% ; p<0.01)$. Emotional expression did not contribute significant variance in the regression equation $(p>0.05$; Step 1 Beta $=0.00)$. As hypothesized, mindfulness-based attention entered at level 2 in the model explained a significant amount of unique variance $(2 \%, \quad p<0.05 ;$ Step 2 ; Beta $=0.15$ ), with greater levels of mindfulness being associated with improved perceptions of physical health status.

In terms of the perceived impact of health on physical functioning, positive affectivity $(p>0.05$; Step 1 Beta $=-0.04)$, negative affectivity $(p>0.05$; Step 1 Beta=-0.09), emotional processing $(p>0.05 ;$ Step 1 Beta $=-0.07)$, and emotional expression $(p>0.05$; Step 1 Beta $=0.03)$ did not account for a significant amount of variance $(1.6 \%$; $p>0.05)$. As hypothesized, mindfulness-based attention entered at level 2 in the model explained a significant amount of unique variance $(7 \%, p=0.001$; Step 2 Beta $=0.27)$, with greater levels of mindfulness being associated with lower perceived impact of health on physical functioning.

For perceived impact of health on the quality of mental functioning, positive affectivity $(p<0.05$; Step 1 Beta $=0.18)$ and negative affectivity $(p<0.001$; Step 1 Beta $=-0.58)$ accounted for a significant amount of variance (Step 1 Variance $=43.8 \%$ ), whereas emotional processing $(p>0.05$; Step 1 Beta $=-0.08)$ and emotional expression $(p>0.05$; Step 1 Beta $=0.02)$ did not. As hypothesized, mindfulness-based attention entered at level 2 in the model explained a significant amount of unique variance $(1.9 \%$, $p<0.05$; Step 2 Beta $=0.14)$, with greater levels of mindfulness being associated with lower perceived impact of health status on the quality of mental functioning.

\section{Discussion}

The purpose of the current investigation was therefore concurrently to examine the unique explanatory value of mindfulness-based attention in relation to theoretically relevant risk and protective factors in regard to both emotional vulnerability (anxiety and depressive symptoms) and perceived health variables (perceptions of health status and physical and mental health impairment). Partially consistent with prediction, mindfulness-based attention incrementally and significantly predicted anhedonic depressive symptoms, but not anxious arousal, over and above the theoretically relevant factors of negative and positive affectivity as well as indices of approachoriented coping. The size of the observed effect for anhedonic depressive symptoms was small in magnitude, at $2.7 \%$. Although the size of this anhedonic depressive effect was relatively small in statistical terms, it is noteworthy that nearly $50 \%$ of total variance in the regression model was accounted for by the step 1 covariates. Furthermore, mindfulnessbased attention shared variance with both positive $(r=0.17)$ and negative affectivity $(r=-0.21)$. Given the magnitude of variance accounted for at step 1 and the shared variance between mindfulness-based attention and positive and negative affectivity, it is noteworthy that mindfulness-based attention enhanced the model's predictive power at all 
(Abelson, 1985). This finding is broadly consistent with mindfulness-based conceptualizations of depressive vulnerability and past empirical work (Teasdale, Segal, \& Williams, 1995; Williams et al., 2000) and extends such findings by demonstrating that associations between mindfulness and depressive symptomatology cannot be attributed solely to shared variance with temperamental or approach-oriented coping factors. Future research should extend the present findings by employing measures that can tap others aspects of depressive vulnerability, such as cognitive (e.g. hopelessness thinking, denial), behavioural (e.g. social withdrawal, disrupted sleeping) and psychophysiological (e.g. reactivity of respiratory sinus arrhythmia, emotional blunting) factors. Such effects, if apparent, would help to rule out the possibility that the present findings are due to shared method variance and cast tests of mindfulness-based attention within a more comprehensive nomological net of evidence. Furthermore, future research could also examine whether the mindfulness-depressive association is due to the theorized protective function of high levels of mindfulness, or a diathesis conferred by low levels of mindfulness.

There was no evidence that mindfulnessbased attention was incrementally related to anxious arousal symptoms beyond theoretical covariates. Such data are inconsistent with past empirical tests of incremental validity using the MAAS specifically (Brown \& Ryan 2003) and the conceptual basis of other anxiety-relevant work generally (Goleman \& Schwartz, 1976; Miller, Fletcher, \& KabatZinn, 1995). This finding may indicate that prior results are attributable to variance in mindfulness-based attention that is shared with other theoretically relevant factors associated with anxious arousal. It is important to note that past tests of the MAAS in relation to anxiety and depressive symptoms utilized assessment devices that do not explicate variance that is unique to these emotional states (i.e. Beck Depression Inventory and Profile of Mood States), as does the MASQ that was employed in the present investigation (Watson et al., 1995). The discordance between anhedonic depressive and anxious arousal symptoms in the current study therefore highlights the utility of using assessment devices that tap unique features of anxiety (e.g. "felt dizzy") and depression (e.g. "felt nothing was enjoyable"), rather than symptoms that are shared between the 2 affective states (e.g. "felt nervous" and "felt discouraged"). Based upon the present data, future work may seek to refine theoretical conceptualizations of the role of mindfulnessbased attention in models of anxiety. For example, vulnerability for anxiety and its disorders may simply be better accounted for by self-regulation factors such as avoidance or tendency to experience negative affectivity than mindfulness-based attention per se (Feldner, Zvolensky, \& Leen-Feldner, 2004). Before more firm conclusions can be drawn about the unique association(s) or lack thereof between mindfulness and anxiety, however, it will be important for future research to independently replicate the present results among other samples using measures that tap mindfulness from an alternative conceptual framework. Additionally, it will be important to utilize alternative dependent measures that reflect aspects of anxiety vulnerability beyond symptoms (e.g. information processing biases, cardiac reactivity) and perhaps employ strategies that can provoke anxiety responses in real time (e.g. biological challenge) to rule out the role of memory or recall biases in the present results.

Regarding the perceived health variables, there was broad-based consistency in the observed effects. Mindfulness-based attention incrementally predicted over and above the temperamental and approach-oriented coping factors perceived global health $(2 \%$ unique variance), perceived impact of health on physical aspects of life functioning ( $7 \%$ unique variance) and perceived impact of health on mental aspects of life functioning $(1.9 \%$ unique variance). These findings are broadly consistent with past theory and research suggesting that mindfulness plays an important role in better understanding physical health processes (Carlson et al., 2003). The present data also conceptually replicate and uniquely extend previous findings using the MAAS, by rigorously establishing that previous mindful-attention effects were probably not due simply to shared variance of mindfulness with other theoretically-relevant factors, and by identifying a similar pattern of results for alternative health-oriented mea- 
sures (Brown \& Ryan, 2003). Thus, the present investigation provides converging evidence for mindfulness-based attention in terms of perceived health processes, with greater levels of mindfulness being associated with less health disability as well as improved ratings of one's health status. These findings should encourage interested researchers to begin theorizing and evaluating the mechanisms underlying such observed effects. There are at least 2 promising avenues to pursue. One is that mindfulness-based attention may increase awareness of bodily oriented processes and prompt more adaptive behaviour (e.g. positive health choices, decreased riskoriented behaviour), thereby leading to more positive perceptions of health. Another nonmutually exclusive pathway may be related to psychological processes in the context of health factors. Specifically, mindfulness-based attention may decrease emotional distress or stress and thereby impact both perceptions of health, physical and mental, as well as healthrelated consequences of chronic emotional distress or stress (Massion et al., 1995). In all cases, future research should build from the present and past work by utilizing a multimethod approach in the assessment of physical health processes.

Moreover, the mindfulness construct has received growing clinical attention in recent years. Although a promising protective factor and target of clinical interventions, few studies have examined the basic processes underlying mindfulness and its associations with emotional processes and outcomes. This type of basic empirical knowledge is, however, essential to translational efforts to conceptualize, advance, and develop cognitive-behavioural strategies that attempt to harness mindfulness skills for the purpose of remedying psychological and health-related problems. This lack of empirical research therefore underscores the relevance of the present study, as 1 step in a nascent systematic program of research focused on mindfulness that promises to inform evolving mindfulness-based cognitivebehavioural therapeutic strategies.

It also is important to comment briefly on the pattern of zero-order associations observed between mindfulness-based attention and the other theoretically relevant predictor variables. The present findings replicated those reported by Brown and
Ryan (2003) in regard to associations between mindfulness-based attention and positive and negative affectivity. Thus, there is increased confidence that MAAS scores are related to a decreased tendency to experience negative affect and an increased tendency to experience positive affect. There also was novel evidence that MAAS scores were not significantly related to either emotional expression or processing modes of approach-oriented coping, as indexed by the well-established EACQ (Stanton et al., 2000). These data provide empirical evidence that mindfulness-based attention is a distinct construct from approach-oriented coping. Future tests may benefit by extending the present results to other coping and affect regulation conceptualizations (e.g. Compas, Connor-Smith, Saltzman, Thomsen, \& Wadsworth, 2001), in order to help rule out alternative conceptualizations of self-regulatory processes in mindfulness-based effects. Similarly, researchers could address associations between mindful attention beyond other theoretically relevant factors, such as distractibility, inattention, or related impulsivity. This work would serve to expand and clarify the empirical associations between mindfulness-based attention and a number of other constructs potentially related to mindfulness-based attention. Similarly, future study could usefully be directed at evaluating whether mindful-attention holds unique explanatory power in relation to related higher-order constructs, such as emotional intelligence.

There are some additional interpretative caveats and directions for future research that warrant consideration. First, although the MASS is a well-developed and useful measure of mindful attention, future study could be directed at evaluating alternative conceptualizations of mindfulness. This type of research would not only provide important data on the nature of specific types of mindfulness being investigated, but also serve to clarify the distinctions between different conceptualizations of this construct. Secondly, the present sample is limited in the sense that it is comprised of a relatively homogenous group of young European American, educated adults recruited from the community, who volunteered to participate in the study for monetary reward. To rule out the possibility that the present results were somehow related 
to a self-selection bias, it will be important for researchers to draw from a more diverse group of persons (e.g. ethnicity, age) as well as utilize recruitment tactics other than those related to community advertisement. Thirdly, we employed a cross-sectional test of the study hypotheses. Future study will need to be extended to naturalistic settings and prospective designs across larger periods of time.

Fourthly, it is important to keep in mind that causal relationships cannot be inferred from the present data. As a next research step, future studies may benefit by experimentally manipulating mindfulness-based attention via differential manipulation in laboratory environments and evaluate corresponding effects on psychological and physical processes. Finally, we examined perceived health processes, as these were the theoretically-derived dependent factors of interest for the present investigation. Future studies could assess both perceived and objective health status, in order to elucidate the role of mindfulness in the intersections between perceived and actual health processes.

\section{Acknowledgements}

This paper was supported by National Institute on Drug Abuse research grants (1 R01 DA018734-01A1, R03 DA16307-01, and 1 R21 DA016227-01) awarded to Dr Zvolensky. This work also was supported by a National Research Service Award (F31 MH073205-01) awarded to Amit Bernstein and a National Research Service Award postdoctoral fellowship (1 F32 MH069037-01A1) awarded to Dr Yartz.

\section{References}

Abelson, R. P. (1985). A variance explanation paradox: when a little is a lot. Psychological Bulletin, 97, 129-133.

Baer, R. A., Smith, G. T., \& Allen, K. B. (2004). Assessment of mindfulness by self-report: The Kentucky Inventory of Mindfulness Skills. Assessment, 11, 191-203.

Bishop, S. R., Lau, M., Shapiro, S., Carlson, L., Anderson, N. D., Carmody, J., Segal, Z. V., Abbey, S., Speca, M., Velting, D., \& Devins, G. (2004). Mindfulness: A proposed operational definition. Clinical Psychology: Science and Practice, 11, 230-241.

Brown, K. W., \& Ryan, R. M. (2003). The benefit of being present: mindfulness and its role in psychological well-being. Journal of Personality and Social Psychology, 84, 822-848.

Buchheld, N., Grossman, P., \& Walach, H. (2002). Measuring mindfulness in insight meditation (Vipassana) and meditation-based psychotherapy: the development of the Frieburg mindfulness inventory. Journal of Meditation Research, 1, 11-34.

Carlson, L. E., Speca, M., Patel, K. D., \& Goodey, E. (2003). Mindfulness-based stress reduction in relation to quality of life, mood, symptoms of stress, and immune parameters in breast and prostate cancer outpatients. Psychosomatic Medicine, 65, 571-581.

Cohen, J., \& Cohen, P. (1983). Applied Multiple Regression/Correlation Analysis for the Behavioral Sciences. Hillsdale, NJ: Erlbaum.

Compas, B. E., Connor-Smith, J. K., Saltzman, H., Thomsen, A. H., \& Wadsworth, M. E. (2001). Coping with stress during childhood and adolescence: problems, progress, and potential in theory and research. Psychological-Bulletin, 127, 87-127.

Conte, H. R., Plutchik, R., Jung, B., \& Picard, S. (1990). Psychological mindedness as a predictor of psychotherapy outcome: a preliminary report. Comprehensive Psychiatry, 31, 426-431.

Demick, J. (2000). Toward a mindful psychological science: theory and application. Journal of Social Issues, 56, 141-159.

Feldner, M. T., Zvolensky, M. J., \& Leen-Feldner, E. (2004). A critical review of the literature on coping and panic disorder. Clinical Psychology Review, 24, 123-148.

Goleman, D. J., \& Schwartz, G. E. (1976). Meditation as an intervention in stress reactivity. Journal of Consulting and Clinical Psychology, 44, 456-466.

Gray, J. A., \& McNaughton, N. (1996). The neuropsychology of anxiety: a reprise. In D. A. Hope (Ed.), Nebraska Symposium on Motivation: Perspectives on Anxiety, Panic, and Fear (Vol. 43, pp. 61-134), Lincoln: University of Nebraska Press.

Gregor, K., Zvolensky, M. J., Leen-Feldner, E. W., Yartz, A. R., \& Feldner, M. T. (in press). Perceived health: a test of incremental validity in relation to smoking outcome expectancies, motivation to smoke, and desire to quit smoking. Cognitive Behaviour Therapy.

Gregor, K., Zvolensky, M. J., \& Yartz, A. R. (2005). Perceived health among individuals with panic disorder: associations with affective vulnerability and psychiatric disability. Journal of Nervous and Mental Disease, 193, 697-699.

Hayes, S. C., \& Wilson, K. G. (2003). Mindfulness as method and process. Clinical Psychology: Science and Practice, 10, 161-165.

Idler, E. L., \& Angel, R. J. (1990). Self-rated health and mortality in the NHANES-I epidemiologic follow-up study. American Journal of Public Health, 80, 446- 452.

Idler, E. L., \& Kasl, S. (1991). Health perceptions and survival: do global evaluations of health status really predict mortality? Journal of Gerontology, 46, S55-S65. 
Kabat-Zinn, J., Lipworth, L., Burney, R., \& Sellers, W. (1986). Four year follow-up of a meditation-based program for the selfregulation of chronic pain: treatment outcomes and compliance. The Clinical Journal of Pain, 2, $159-173$.

Langer, E. J. (1989). Mindfulness. Reading: Addison Wesley Longman.

Langer, E. J., \& Moldoveneanu, M. (2000). Mindfulness research and the future. Journal of Social Issues, 56, 129-140.

Massion, A. O., Teas, J., Hebert, J. R., Wertheimer, M. D., \& Kabat-Zinn, J. (1995). Meditation, melatonin, and breast/prostate cancer: hypothesis and preliminary data. Medical Hypotheses, 44, 39-46.

Miller, J. J., Fletcher, K., \& Kabat-Zinn, J. (1995). Three-year follow-up and clinical implications of a mindfulness meditation-based stress reduction intervention in the treatment of anxiety disorders. General Hospital Psychiatry, 17, 192-200.

Mossey, J. M., \& Shapiro, E. (1982). Self-rated health: a predictor of mortality among the elderly. American Journal of Public Health, 71, 800-808.

Reibel, D. K., Greeson, J. M., Brainard, G. C., \& Rosenzweig, S. (2001). Mindfulness-based stress reduction and health-related quality of life in a heterogeneous patient population. General Hospital Psychiatry, 23, 183-192.

Roemer, L., \& Orsillo, S. M. (2002). Expanding our conceptualization of and treatment for generalized anxiety disorder: integrating mindfulness/ acceptance-based approaches with existing cognitive-behavioral models. Clinical Psychology: Science \& Practice, 9, 54-68.

Schmidt, N. B., Joiner, T. E., Staab, J. P., \& Williams, F. M. (2003). Health perceptions and anxiety sensitivity in patients with panic disorder. Journal of Psychopathology and Behavioral Assessment, 25, 139-145.

Schmidt, N. B., \& Telch, M. J. (1997). Nonpsychiatric medical comorbidity, health perceptions, and treatment outcome in patients with panic disorder. Health Psychology, 16, 114-122.

Schmidt, N. B., Telch, M. J., \& Joiner, T. E. (1996). Factors influencing health perceptions in patients with panic disorder. Comprehensive Psychiatry, 37, 253-260.

Smyth, J., \& Pennebaker, J. (1999). Telling one's story: translating emotional experiences into words as a coping tool. In C. R. Snyder (Ed.), Coping: The Psychology of What Works (pp. 70-89). New York: Oxford University Press.

Stanton, A. L., Kirk, S. B., Cameron, C. L., \& Danoff-Burg, S. (2000). Coping through emotional approach: scale construction and validation. Journal of Personality and Social Psychology, 78, 1150-1169.

State of Vermont Department of Health, (2000). Retrieved September 3, 2002, from http:// www.healthyvermonters.info/.

Stewart, A. L., Hays, R. D., \& Ware, J. E. Jr. (1988). The MOS short-form General Health Survey. Medical Care, 26, 724-735.

Teasdale, J. D., Segal, Z., \& Williams, J. M. (1995). How does cognitive therapy prevent depressive relapse and why should attentional control (mindfulness) training help? Behaviour Research and Therapy, 33, 25-39.

Watson, D. (2000). Mood and Temperament. New York: Guilford Press.

Watson, D., Clark, L. A., \& Tellegen, A. (1988). Development and validation of brief measures of positive and negative affect: the PANAS scales. Journal of Personality and Social Psychology, 54, 1063-1070.

Watson, D., David, J. P., \& Suls, J. (1999). Personality, affectivity and coping. In C. R. Snyder (Ed.), Coping: The Psychology of What Works. New York: Oxford University Press.

Watson, D., Weber, K., Assenheimer, J. S., Clark, L. A., Strauss, M. E., \& McCormick, R. A. (1995). Testing a tripartite model: I. Evaluating the convergent and discriminant validity of anxiety and depression symptom scales. Journal of Abnormal Psychology, 104, 3-14.

Wilber, K. (2000). Integral Psychology: Consciousness, Spirit, Psychology, Therapy. Boston: Shambhala.

Williams, J., Teasdale, J. D., Segal, Z. V., \& Soulsby, J. (2000). Mindfulness-based cognitive therapy reduces overgeneral autobiographical memory in formerly depressed patients. Journal of Abnormal Psychology, 109, 150-155.

Yartz, A. R., Zvolensky, M. J., Gregor, K., Feldner, M. T., \& Leen-Feldner, E. (2005). Health perception is a unique predictor of anxiety symptoms in nonclinical participants. Cognitive Behaviour Therapy, 34, 65-74.

Zvolensky, M. J., Feldner, M. T., Leen-Feldner, E., \& Yartz, A. R. (2005). Exploring basic processes underlying acceptance and mindfulness. In S. Orsillo, L. Roemer, (Eds), Acceptance and Mindfulness-Based Approaches to Anxiety: Conceptualization and Treatment (pp. 325-359). New York: Springer.

Zvolensky, M. J., Kotov, R., Antipova, A. V., \& Schmidt, N. B. (2005). Diathesis stress model for panic-related distress: a test in a Russian epidemiological sample. Behaviour Research \& Therapy, 43, 521-532. 
Copyright of Cognitive Behaviour Therapy is the property of Routledge and its content may not be copied or emailed to multiple sites or posted to a listserv without the copyright holder's express written permission. However, users may print, download, or email articles for individual use. 